\title{
PROPERTIES OF COSMIC SHOCK WAVES IN LARGE-SCALE STRUCTURE FORMATION
}

\author{
Francesco Miniati, ${ }^{1}$ Dongsu Ryu, ${ }^{2}$ Hyesung Kang, ${ }^{3}$ T. W. Jones, ${ }^{1}$ Renyue Cen, ${ }^{4}$ and Jeremiah P. Ostriker ${ }^{4}$ \\ Received 1999 December 12; accepted 2000 May 30
}

\begin{abstract}
We have examined the properties of shock waves in simulations of large-scale structure formation. Two cosmological scenarios have been considered: a standard cold dark matter model with $\Omega_{M}=1$ (SCDM), and a cold dark matter model with cosmological constant and $\Omega_{M}+\Omega_{\Lambda}=1(\Lambda \mathrm{CDM})$ having $\Omega_{\Lambda}=0.55$. Large-scale shocks result from accretion onto sheets, filaments, and knots of mass distribution on a scale of the order of $\sim 5 h^{-1} \mathrm{Mpc}$ in both scenarios. Energetic motions, partly residuals of past accretion processes and partly caused by current asymmetric inflow along filaments, end up generating additional shocks. These extend on a scale of the order of $\sim 1 h^{-1} \mathrm{Mpc}$ and envelop and penetrate deep inside the clusters. Collisions between substructures inside clusters also form merger shocks. Consequently, the topology of the shocks is very complex and highly connected. During cosmic evolution the comoving shock surface density decreases, reflecting the ongoing structure merger process in both scenarios. Accretion shocks have very high Mach numbers, typically between 10 and a few $\times 10^{3}$, when photoheating of the preshock gas is not included. The characteristic shock velocity is of the order of $v_{\mathrm{sh}}(z)=$ $H(z) \lambda_{\mathrm{nl}}(z)$, where $\lambda_{\mathrm{nl}}(z)$ is the wavelength scale of the nonlinear perturbation at the given epoch. However, the Mach number for merger and flow shocks (which occur within clusters) is usually smaller, in the range of $\sim 3-10$, corresponding to the fact that the intracluster gas is hot (i.e., already shock heated). Statistical fits of shock velocities around clusters as a function of cluster temperature give power-law functions in accord with those predicted by one-dimensional solutions. On the other hand, a very different result is obtained for the shock radius, reflecting extremely complex shock structures surrounding clusters of galaxies in three-dimensional simulations. The amount of inflowing kinetic energy across the shocks around clusters, which represents the power available for cosmic-ray acceleration, is comparable to the cluster X-ray luminosity emitted from a central region of radius $0.5 h^{-1} \mathrm{Mpc}$. Considering their large size and long lifetimes, those shocks are potentially interesting sites for cosmic-ray acceleration, if modest magnetic fields exist within them.
\end{abstract}

Subject headings: acceleration of particles — galaxies: clusters: general -

large-scale structure of universe - methods: numerical - shock waves -

$\mathrm{X}$-rays: galaxies

\section{INTRODUCTION}

Cosmic shock waves, formed in the course of large-scale structure formation, can play important roles in cosmology. They include external accretion shocks, as well as merger and flow shocks internal to galaxy clusters. The pristine cosmic plasma accreting onto the large-scale structure is deflected from the Hubble flow and first processed by accretion shocks (see, e.g., Ryu \& Kang 1997; hereafter RK97). Evidence for their existence might be inferred from the observation of hot gas in the intracluster medium (ICM). In the commonly accepted paradigm for structure formation in the universe, gas accreting onto cosmic filaments and clusters of galaxies (GCs) has a typical bulk velocity up to $\sim$ a few $10^{3} \mathrm{~km} \mathrm{~s}^{-1}$. This gas is then shock heated to temperatures ranging from $10^{5}$ to $10^{7} \mathrm{~K}$ in filaments and up to $10^{7}-10^{8} \mathrm{~K}$ in GCs (e.g., Kang et al. 1994a, hereafter KCOR94; Cen \& Ostriker 1994, hereafter CO94; Cen \& Ostriker 1999a). Merger shocks are produced during

\footnotetext{
${ }^{1}$ School of Physics and Astronomy, University of Minnesota, Minneapolis, MN 55455.

${ }^{2}$ Department of Astronomy and Space Science, Chungnam National University, Daejeon 305-764, Korea.

${ }^{3}$ Department of Earth Science, Pusan National University, Pusan 609735 , Korea.

${ }^{4}$ Princeton University Observatory, Princeton, NJ 08544.
}

mergers of substructures within a galaxy cluster and propagate through the hot ICM. During such a process accretion shocks associated with the merging units also propagate through the ICM. Together with merging shocks, they form a complex structure that can survive for long times inside the ICM after the end of the merger because of the continuous gas inflow through filaments and sheets. We refer to these as "flow shocks" (see $\S 3.1$ ). There is now substantial observational evidence for temperature structure in clusters due to internal shock waves; these appear to be mostly produced by merger events (e.g., Markevitch et al. 1998; Donnelly et al. 1999 and references therein), although recently there may also be some evidence for flow shocks as well (Ensslin et al. 1998, § 4).

Among other reasons for interest in cosmic shocks is their ability to efficiently accelerate particles to relativistic energies (cf. Blandford \& Ostriker 1978, 1980; see also Jones \& Ellison 1991 for a recent review of this subject). In fact, relativistic cosmic-ray (CR) electrons have been observed in GCs through their synchrotron emission (e.g., Kim et al. 1989; Giovannini et al. 1993; Deiss et al. 1997). Extended sources of synchrotron radiation are commonly observed with spatial distribution similar to that of the thermal X-ray emission (see, e.g., Liang 1999). Although the cooling time for such CR electrons is much shorter than the cluster ages, explicit signatures of particle aging are rare in the spectra of 
the observed sources. Since individual cluster galaxies are unlikely to replenish the ICM adequately with populations of relativistic particles, an efficient mechanism for extended particle acceleration is probably required to understand the CR electron replenishment.

Moreover, recently the Extreme Ultraviolet Explorer $(E U V E)$ satellite has revealed that many clusters possess an excess of extreme-ultraviolet (EUV) radiation compared to what is expected from the hot, thermal X-ray-emitting ICM (e.g., Lieu et al. 1996; Fabian 1996; Mittaz, Lieu, \& Lockman 1998; Kaastra 1998). Further evidence for nonthermal activity in the ICM comes from the detection of radiation in excess of thermal emission in the hard X-ray band above $\sim 10 \mathrm{keV}$ (e.g., Henriksen 1998; FuscoFemiano et al. 1999; Valinia et al. 1999; Sarazin 1999). The mechanism proposed for the origin of these components is the inverse-Compton scattering of cosmic microwave background photons by CR electrons, although it is not clear whether the same electron population is responsible for producing both the EUV excess and the hard X-ray excess (Ensslin, Lieu, \& Biermann 1999). Such detections suggest the possibility that nonthermal activities in the ICM are much higher than previously expected (Sarazin \& Lieu 1998; Lieu et al. 1999).

Cosmic shock waves should be capable of accelerating $\mathrm{CR}$ electrons responsible for the above emissions. However, at the same time, CR protons are produced. It is then possible, although not yet established, that if the above interpretation of the nonthermal radiation is correct, the CR protons produced at these shocks and accumulated throughout the cosmological evolution could provide a substantial fraction of the total pressure in GCs (Sarazin \& Lieu 1998; Lieu et al. 1999). It is clear that if the CR pressure was ever comparable to the thermal pressure during the evolution of the universe, this would have a profound impact on cosmology. For instance, structure formation is heavily used as a probe for discriminating among cosmological models (e.g., Carlberg et al. 1997; Bahcall \& Fan 1998), and hydrostatic equilibrium of the thermal ICM gas in the potential well of the total cluster mass is commonly assumed in order to derive GC masses (e.g., White, Efstathiou, \& Frenk 1993; Evrard 1997). The presence of a nonthermal component obviously would alter the results in proportion to its relative importance (Ensslin et al. 1997). Furthermore, some additional source of pressure is clearly required in GCs over that produced by adiabatic hydrodynamics, both to produce the correct density profiles (e.g., Evrard 1990; Navarro, Frenk, \& White 1995) and to prevent catastrophic cooling flows (Suginohara \& Ostriker 1998). CR pressure in the inner parts of GCs may thus play a vital role in the hydrodynamic equilibrium of these systems.

Accretion shocks have also been proposed as sites for the acceleration of high-energy cosmic rays, protons, and heavy nuclei, up to $10^{18}-10^{19} \mathrm{eV}$ (Kang, Ryu, \& Jones 1996; Kang, Rachen, \& Biermann 1997). In fact, given the large velocity of the accretion flows and the large size and long lifetimes of the associated shocks, such energies would be achievable through "cross-field" diffusion in perpendicular magnetohydrodynamic shocks. Magnetic fields in the ICM of GCs have been observed with strengths of the order of a few $\times 0.1 \mu \mathrm{G}$ (e.g., Kim et al. 1989; Fusco-Femiano et al. 1999; Molendi et al. 1999). Outside GCs, constraints on rotational measure from quasars impose an upper limit of $\sim \mathrm{nG}$, based on the assumption of a regularly alternating magnetic field (see, e.g., Kronberg 1994). However, this limit can be shifted to higher values if a realistic distribution of magnetic fields associated with cosmic structures is assumed. On such a basis, Ryu, Kang, \& Biermann (1998) and Blasi, Burles, \& Olinto (1999) claimed a new upper limit of $\lesssim 1 \mu \mathrm{G}$, at least along cosmic structures.

Cosmic shock waves could serve also as sites for the generation of weak seeds of magnetic field by the Biermann battery mechanism. It has been proposed that these seeds could be amplified to strong magnetic fields of up to $\sim \mu \mathrm{G}$ in clusters if flows there can be described as Kolmogorov turbulence (Kulsrud et al. 1997). However, further development into coherent magnetic fields is unclear, since there is as yet no detailed theory capable of describing this process (see, e.g., Chandran 1997).

Additional roles that shock waves may play in cosmology have been explored by a number of authors (e.g., RK97; Quilis, Ibanez, \& Saez 1998). RK97 compared analytical self-similar solutions for cluster formation in the Einsteinde Sitter universe (Bertschinger 1985) with one-dimensional numerical simulations in low-density universes with and without a cosmological constant $\left(0.1<\Omega_{M}<1\right)$, where the properties of the accretion flow are related to the cluster's mass, radius, and temperature. The major conclusion was a possibly testable prediction about the difference in the accretion flow in different cosmological models. In particular, the accretion velocity onto clusters of a given mass or radius in low-density universes is smaller by up to $45 \%$ or $65 \%$, respectively, compared to that in an Einstein-de Sitter universe.

In the present paper we focus on the quantitative properties of large-scale shocks produced by gas during the formation of cosmic structures. For this, the simulation data described in KCOR94 and CO94 have been used. The roles played by those shocks, especially with regard to CR acceleration and magnetic field generation and their consequences for cosmology, will be studied in future works. Details of our data analysis are described in $\S 2.2$. In particular, we computed the velocity, Mach number, and radius of shocks and the kinetic energy flow across them, which have not been studied in previous works, and calculated their correlation with the cluster core temperature. The results are reported in $\S 3$. Finally, $\S 4$ concludes with a discussion.

\section{NUMERICS}

\subsection{Simulations and Data}

We study the properties of shock waves associated with the large-scale structure of the universe in numerical simulations. In particular, we investigate the velocity and radius of shocks in terms of cluster X-ray temperatures. The latter, in fact, is the most reliably reproducible quantity in numerical simulations (e.g., Kang et al. 1994b; Frenk et al. 1999) and has recently been measured with satellite observations (e.g., Arnaud 1994; Mushotzky \& Scharf 1997).

Simulation data in two cosmological scenarios have been used: a standard CDM (SCDM) model and a CDM $+\Lambda$ $(\Lambda \mathrm{CDM})$ model. The SCDM simulation was discussed by KCOR94 and is characterized by the following parameters: spectral index for the initial power spectrum of perturbations $n=1$, normalized Hubble constant $h \equiv H_{0} /(100$ $\left.\mathrm{km} \mathrm{s}^{-1} \mathrm{Mpc}^{-1}\right)=0.5$, total mass density $\Omega_{M}=1$, baryonic 
fraction $\Omega_{b}=0.06$, and normalization $\sigma_{8}=1.05$. On the other hand, the $\Lambda$ CDM simulation was discussed by $\mathrm{CO} 94$ and featured the following parameters: $n=1, h=0.6$, $\Omega_{M}=0.45, \Omega_{\Lambda}=0.55\left(\Omega_{0}=\Omega_{M}+\Omega_{\Lambda}=1\right), \Omega_{b}=0.043$, and $\sigma_{8}=0.77$. In both calculations a cubic region of size 85 $h^{-1} \mathrm{Mpc}$ at the current epoch was simulated inside a computational box with $270^{3}$ cells and $135^{3}$ dark matter (DM) particles, allowing a spatial resolution of $0.315 h^{-1} \mathrm{Mpc}$. We refer the reader to KCOR94 and CO94 for further details. The simulations were performed with the code described in Ryu et al. (1993). In addition to DM, the evolution of gas was followed with a grid-based hydrodynamic code.

\subsection{Analysis Method}

A crucial and lengthy part of the current analysis of the results is the extraction and interpretation of detailed information from the numerical data. Each simulation forms thousands of clusters and a very rich system of associated shock waves. We outline our methods here.

\subsubsection{Cluster Identification and DM Related Properties}

The first step to take is the identification of GCs in the data. This could be done either through the X-ray emissivity criterion detailed in KCOR94 or through the distribution of DM particles. The former is preferred when the derived quantities must be directly compared with observational tests. However, when studying dynamical properties of GCs such as accretion velocity onto them, it would be better to use DM particles for identification, because it is mainly the gravitational contribution of this component that determines those properties. Therefore, for the identification of GCs we have adopted the DM-based "spherical overdensity" method described in Lacey \& Cole (1994), with a slight modification to speed up the calculation process. In particular, each DM particle is placed inside a cell [of physical size $\left(0.315 \mathrm{~h}^{-1} \mathrm{Mpc}\right)^{3}$ ] of the full $270^{3}$ grid according to its position. If the number of particles inside such a cell exceeds a given threshold $N_{\text {threshold }}$, then for each particle location we define a local number density $n_{l}=$ $3(N+1) /\left(4 \pi R_{N}^{3}\right)$, where $R_{N}$ is the distance to the $N$ th nearest neighbor. We then take the highest density particle locations as the candidate centers of clusters. The "candidate" particle locations are then sorted by density and a sphere is grown around each one of them, with the radius being increased until the mean density decreases to a value $\delta \bar{\rho}$, where $\delta$ is a selected parameter and $\bar{\rho}=\Omega_{M} \rho_{\text {crit }}$ is the mean background density of the universe. The center of mass (CM) of the particles inside the sphere is calculated and taken as a new center, and the overall process is iterated until center corrections are smaller than $\epsilon / n_{l}^{1 / 3}$, where $\epsilon$ is a small parameter. Once the cluster radii, $R_{\delta}$, are thus defined, we reject from our list the smaller of any two clusters whose spatial separation is shorter than $3 / 4$ the sum of their radii. For our analysis we choose $N_{\text {threshold }}=50$; as in Lacey \& Cole (1994), we set $N=10$ and $\epsilon=0.1$. We chose $\delta=80$, since then $\delta \rho$ is about the average density inside the first caustic around clusters in SCDM (Bertschinger 1985; RK97). Thus, the cluster radius is defined by $R_{80}$.

We point out for comparison that we have also identified GCs with the X-ray luminosity method outlined in KCOR94. In general, we have found that the relations between various physical quantities are not affected significantly by the method used. However, since the criteria are different, the samples considered are not identical, with differences depending on the thresholds used.

\subsubsection{Cluster Baryonic Matter Related Quantities}

Once the cluster centers and radii, $R_{80}$, have been defined, we turn to determining their X-ray-based temperature, $T_{\mathrm{X}}$, and luminosity, $L_{\mathrm{X}}$; in addition, we compute the shock radius and velocity, $R_{s}$ and $v_{s}$, respectively, around clusters. The cluster temperature, $T_{\mathrm{X}}$, is computed by averaging over the cluster core region defined by $r<$ $R_{\mathrm{avg}}=0.5 h^{-1} \mathrm{Mpc}$. The X-ray luminosity, $L_{\mathrm{X}}$, however, is averaged over a volume of $r<R_{\text {avg }}=1.0 h^{-1} \mathrm{Mpc}$, since bremsstrahlung emissivity, $j_{\mathrm{ff}}(v)$ (KCOR94, eq. [2]) is substantial up to a distance of $\sim 1 h^{-1} \mathrm{Mpc}$. Operationally, the average is contributed from all the cells falling inside a sphere of volume $V$ (around the cluster), with a weight function, $w$, given by the cell intersection with $V$. So, for example, $w=1$ for central cells and $w \ll 1$ for the marginal ones. This detail is important, because of the rapid drop of the thermodynamic variables with distance from the cluster center. Altering the "natural" weight of the central cells, unless motivated by a corrective purpose, introduces a source of error in the calculated quantities. While our temperature metrics should be fairly accurate, we expect, on the basis of resolution studies (Cen \& Ostriker 1999b), that the $\mathrm{X}$-ray luminosities are systematically underestimated, while the rank order of the luminosities will be correct.

Once the cluster luminosity has been determined, we further select our sample based on the criterion $L_{\mathrm{X}} \geq L_{\mathrm{ff}}=$ $10^{41}$ ergs s$^{-1}$. We note that given the different numerical schemes for the evolution of the baryonic and DM-related variables, the position of the X-ray luminosity peak and the DM-based CM of the same cluster may not correspond exactly. The shift is limited to one grid cell for the highluminosity clusters, but can amount a few cells for the faint ones. Our combined choice of $N_{\text {threshold }}$ and a limiting luminosity assures that the luminosity peak and DM-based CM do not differ by more than 1 grid cell.

Finally, in order to determine the shock radius, $R_{s}$, and velocity, $v_{s}$, we have labeled those cells where shocks are located. Shocks have been identified as compression regions $(\nabla \cdot v<0)$ meeting the following requirements for pressure $(P)$ and velocity $(v)$ jumps (where indexes 1 and 2 refer to preshock and postshock quantities, respectively):

$$
\begin{gathered}
\frac{P_{2}-P_{1}}{P_{1}}>1.5, \\
v_{1}-v_{2}=2 \frac{c_{1}}{\gamma+1} \frac{M_{1}^{2}-1}{M_{1}}>0.87 c_{1},
\end{gathered}
$$

where $c_{1}$ is the preshock sound speed. These criteria select shocks with Mach number $M_{1}>\sqrt{3}$. However, once shocks have been identified with this method, we adopt the temperature jump (across the shock) instead of the pressure in order to calculate their properties. In fact, when a shock is only a few computational cells apart from a cluster center, compression in excess of that produced by the shock wave can result from the gravitational potential of the cluster itself. Given a finite numerical resolution, it then becomes impossible to separate the two effects (due to shock and gravitational compression). However, since the cluster core is thought to be rather isothermal (Evrard, Metzler, \& Navarro 1996), adiabatic gravitational compression affects density, pressure, and velocity substantially more than the 
temperature. Therefore, for cosmological simulations, shock properties based on the temperature jump are the most reliable. Our tests clearly support this point.

The condition enforced by equation (2) does not add any physical property to the selected shocks, with respect to equation (1). However, it is of great advantage in our numerical effort to rule out compression regions simply due to the hydrostatic equilibrium inside the cluster gravitational potential, and otherwise detectable by equation (1) as shocked cells. This scheme has proved successful in tests with shocks of known hydrodynamic properties for several different applications. Once the shocks have been properly identified, the shock radius, $R_{s}$, is calculated by taking the average of the six distances to the first "shocked" cells encountered along the coordinate directions $(x, y$, and $z)$ from the cluster center. In addition, the normal component of the preshock velocity is calculated using the hydrodynamic shock-jump conditions (e.g., Landau \& Lifshitz 1997). We note that the normal component of the shock velocity, $v_{n 1}$, and not $v_{1}$, is really the quantity of interest here. Therefore, in the following discussion this is the quantity we refer to as the shock velocity, $v_{s}$. In fact, it determines the shock Mach number and flux of the kinetic energy available for particle acceleration, the parameters most relevant for the production of CR populations.

\section{RESULTS}

\subsection{Morphological Structure of Cosmic Shock Waves}

Figures 1 and 2 illustrate typical structures found in the simulations. They represent slices of $0.315 h^{-1} \mathrm{Mpc}$ thickness showing bremsstrahlung emission (gray scale) with superposed contours of the shock compression factor $(\nabla \cdot v)$. In both cases, shocks confine filamentary structures where the temperature can vary from $10^{4}$ to $10^{7} \mathrm{~K}$. The most interesting feature, however, is the complexity of shocks around GCs. In both cosmological models, in fact, the accretion flow develops multiple shocks extending over a region of 5-10 $h^{-1} \mathrm{Mpc}$ size around the GCs. This is a generic feature of accretion flows there, and it is directly related to the hierarchical process for cluster formation. In fact, the merging of two substructures into a single unit produces at least two main effects related to shocks. First, shock waves of low Mach number are generated in the collision of the clusters' ICMs, which are commonly referred as merger shocks. However, in addition, part of the accretion shocks previously associated with each substructure end up propagating through the ICM of the newly formed structure, reaching deep inside the cluster core. These shocks, which we refer to as "flow shocks," are subsequently "fed" by residual gas motions in the ICM and

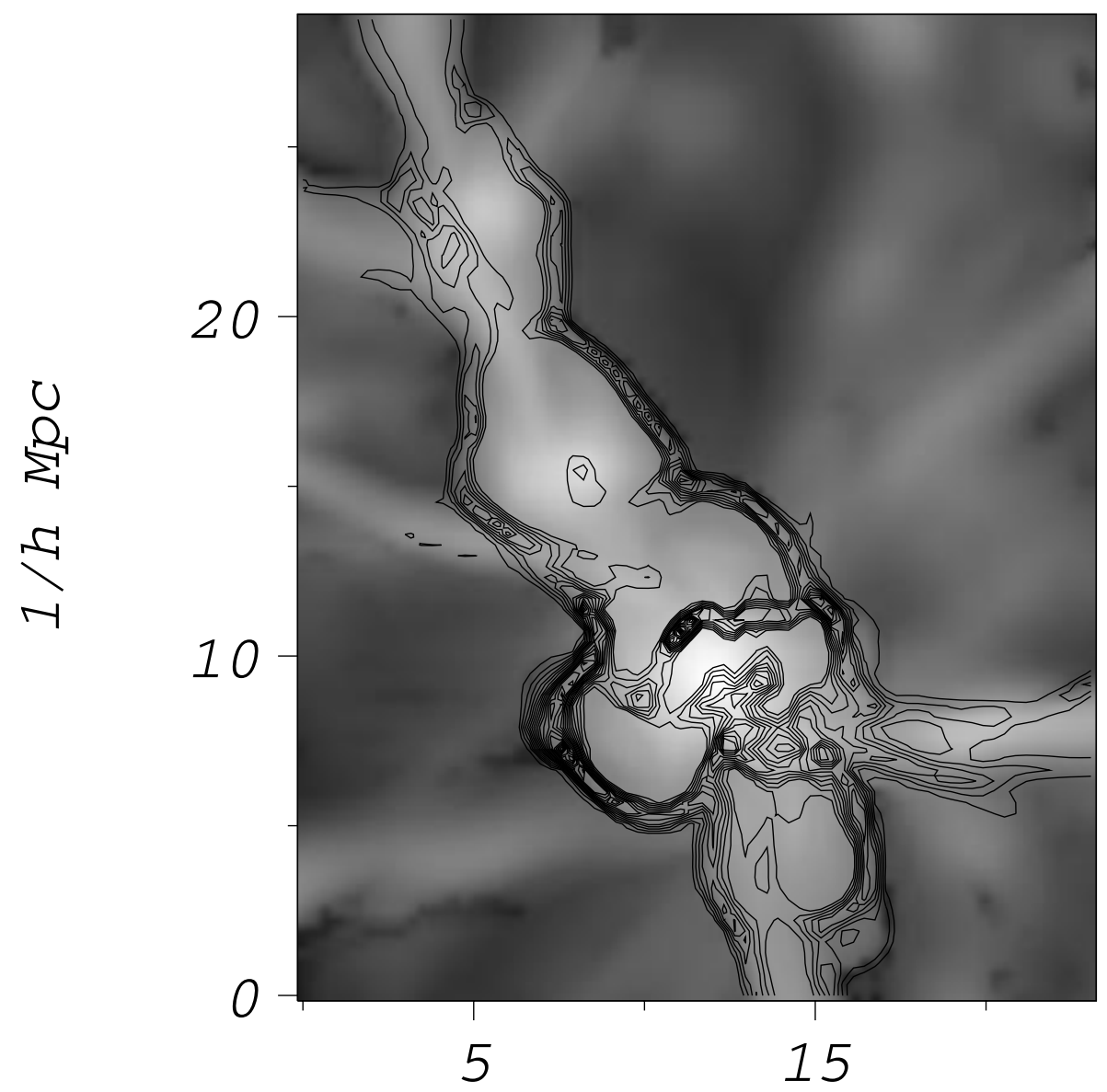

1/h Mpc

FIG. 1.-Typical cosmological structure. Bremsstrahlung X-ray emission (gray scale) superposed on contours of the compression factor of shock waves $(\nabla \cdot v)$ in SCDM. 


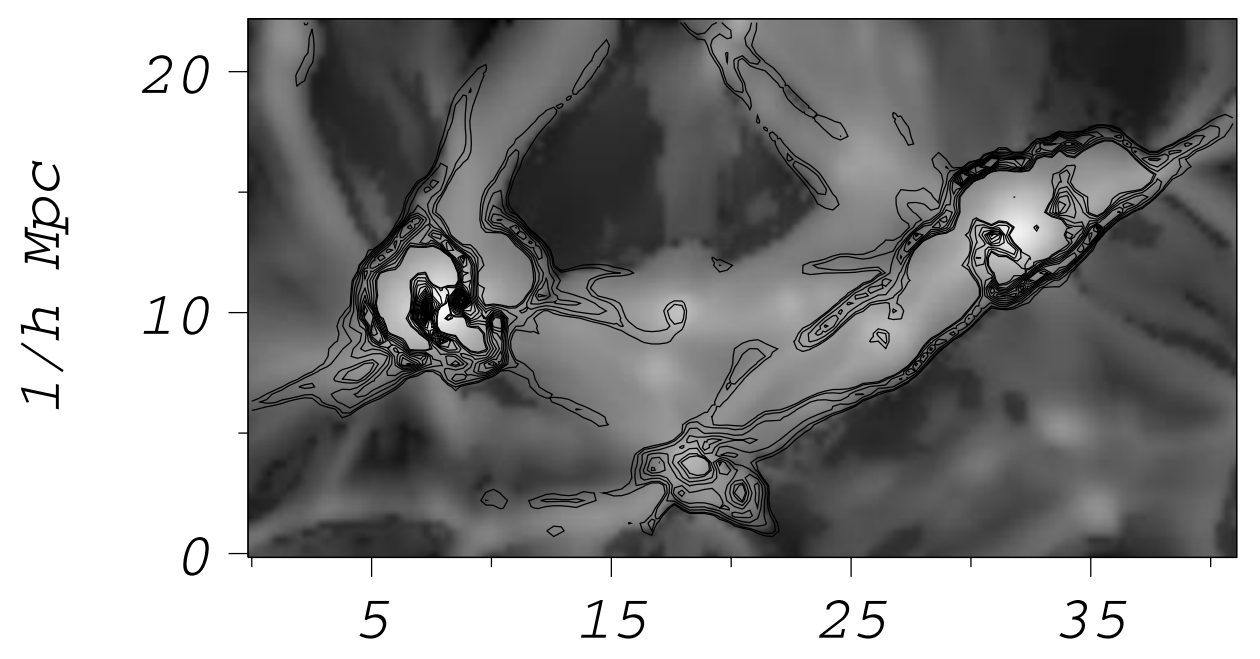

1/h Mpc

FIG. 2.-Same as Fig. 1, but for $\Lambda \mathrm{CDM}$

ongoing gas inflow accreting along filaments and sheets. Their presence, in addition to outer accretion shocks, provides additional heating of the ICM and makes its thermal structure not quite uniform over a region of several $h^{-1}$ $\mathrm{Mpc}$ of size. As already pointed out in $\S 1$, Markevitch et al. (1998, and references therein) and Donnelly et al. (1999, and references therein) showed evidence for significant temperature structures inside clusters. They attribute these to the presence of shocks associated with merger events. However, in some cases the merging is only inferred from the temperature map; in other cases, temperature asymmetry is observed when the merger is just beginning to take place and therefore has not yet been able to affect the cluster temperature structure. In future, cluster shocks could be identified independently of the presence of merging processes. Some of this evidence may actually already be available (Ensslin et al. 1998; see $\S 4$ for further discussion).

A three-dimensional perspective of shocks around GCs in SCDM is offered in Figure 3. This is a combination of shock strength isosurfaces (Fig. 3a) and volume rendering of bremsstrahlung emission (Fig. $3 b$ ) for a portion of the computational domain of size $30 \times 40 \times 30\left(h^{-1} \mathrm{Mpc}\right)^{3}$ at $z=0$. With the help of the bremsstrahlung emission, which identifies the GCs, one can locate shocks in their appropriate cosmological context. This image reveals a further degree of complexity of real cosmic shocks with respect to the two-dimensional slices above. Namely, in addition to being multiple associations with individual clusters, such shocks are also largely connected topologically with neighboring structures. Their shapes, far from spherical, extend over tens of $\mathrm{Mpc}$, forming a continuum that envelops all nearby clusters.

In conclusion, the hierarchical process for structure formation produces an extremely complex shock structure around clusters and groups of clusters (or superclusters). These shock waves are neither spherical nor identifiable by a simple surface. Indeed, they intersect each other, forming nested shock surfaces, penetrating inside the ICM of individual clusters.

\subsection{Physical Relations for Accretion Shock Waves}

In this section we aim for a more quantitative description of accretion flows and shocks. Figure 4 includes four histograms showing the distribution, at redshift zero, of GCs $[N(\mathrm{GC})]$ with respect to the Mach number $(\boldsymbol{M})$ of the associated "external" accretion shocks (top) and "internal" shocks (bottom). The latter have been selected inside regions of radius $0.5 h^{-1} \mathrm{Mpc}$ from the cluster centers. The results are plotted for the SCDM (left) and $\Lambda$ CDM models (right). Mach numbers associated with external accretion shocks are remarkably large, ranging from $\sim 10$ up to a few $\times 10^{3}$ in both cosmologies. We note that this is partly because photoheating of preshock gas by metagalactic radiation field was not included. In addition, feedback processes from massive stars in galaxies may raise the temperature in the outskirts of clusters further. Without these sources of heating, the preshock gas stays cool, with sound speed less than $\sim 1 \mathrm{~km} \mathrm{~s}^{-1}$. If photoheating were properly included, however, the preshock gas temperature would have been raised to $\sim 10^{4} \mathrm{~K}$, with corresponding sound speed $\sim 30$ $\mathrm{km} \mathrm{s}^{-1}$ (see, e.g., Ostriker \& Cen 1996; Cen \& Ostriker 1999a). Hence, the Mach numbers should be significantly smaller than those in Figure 4, yet they would still reach up to $\sim 100$ for external accretion shocks. On the other hand, the Mach numbers associated with internal shocks are much smaller, mostly in the range $\sim 3-10$, peaking at about 5 . It thus emerges that, because internal shocks propagate through a significantly hotter medium, with typically $T \sim 10^{6}-10^{7} \mathrm{~K}$, the velocities associated with both external and internal shocks are comparable.

Among the characteristics of accretion flows onto GCs, the most relevant quantities are the shock velocity, $v_{s}$, and radius, $R_{s}$. These are plotted as a function of each cluster's X-ray temperature in Figure 5, again for SCDM (left) and $\Lambda \mathrm{CDM}$ (right). According to the self-similar solution of onedimensional spherical accretion in the SCDM universe, the cluster is confined by an accretion shock at $R_{s}$, and the temperature of the cluster gas increases inward 


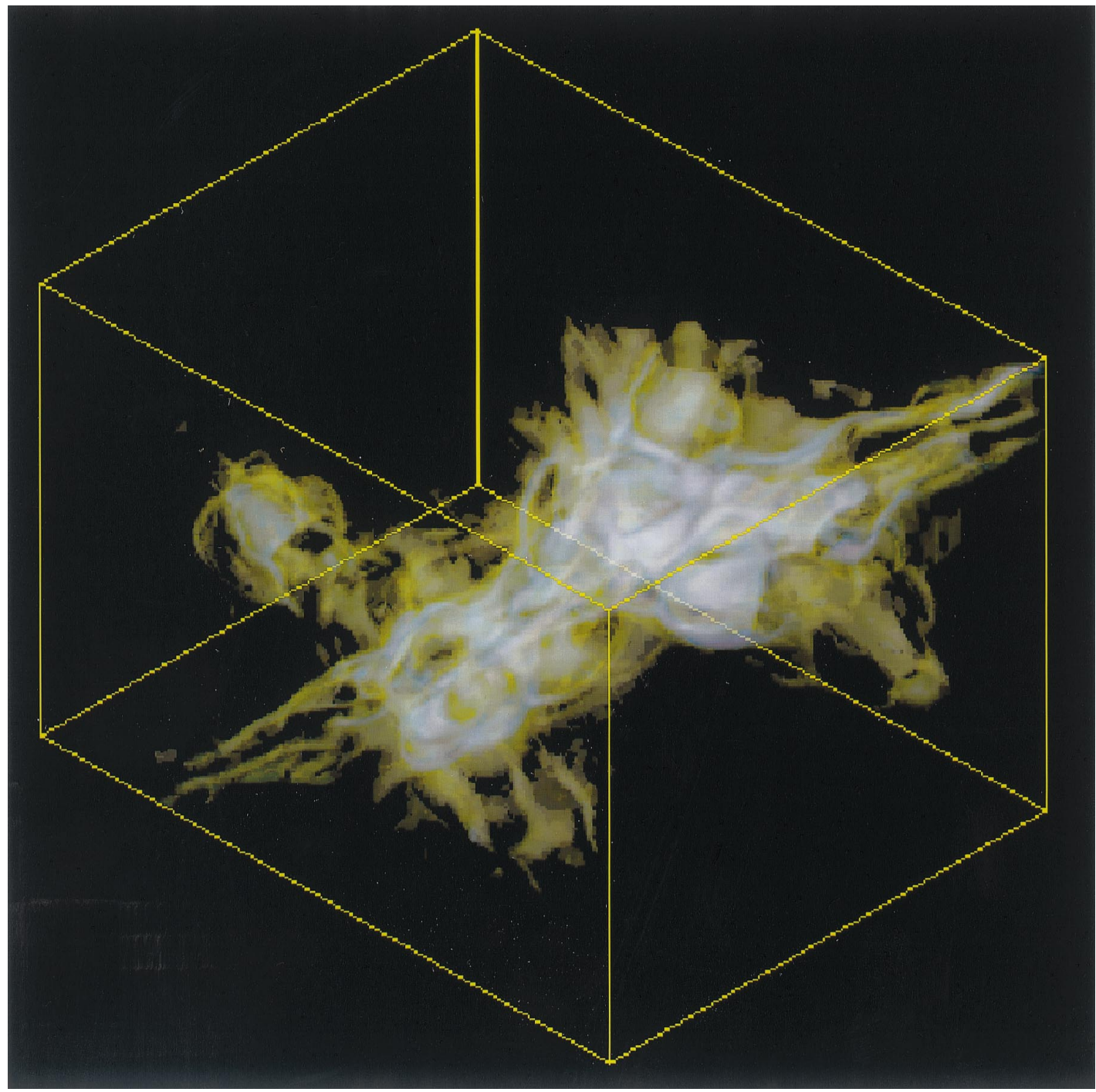

FIG. $3 a$

FIG. 3. - (a) Three-dimensional shock surfaces and (b) volume rendering of bremsstrahlung X-ray emission for a SCDM model simulation. The colors have been assigned according to a rainbow-type color map, where high values correspond to blue/violet and low ones to red. The displayed frame includes a portion of the computational box of size $30 \times 40 \times 30\left(h^{-1} \mathrm{Mpc}\right)^{3}$ at $z=0$.

(Bertschinger 1985; RK97). By choosing the gas temperature at $r=0.3 R_{s}$ as a representative value for the cluster's temperature, $T_{\mathrm{X}}$, the following relations of $v_{s}$ versus $T_{\mathrm{X}}$ and $R_{s}$ versus $T_{\mathrm{X}}$ are expected:

$$
\begin{gathered}
v_{s}=1.75 \times 10^{3} \mathrm{~km} \mathrm{~s}^{-1}\left(\frac{T_{\mathrm{X}}}{7.8 \times 10^{7} \mathrm{~K}}\right)^{1 / 2} \\
R_{s}=2.12 h^{-1} \mathrm{Mpc}\left(\frac{T_{\mathrm{X}}}{7.8 \times 10^{7} \mathrm{~K}}\right)^{1 / 2} .
\end{gathered}
$$

By fitting our data for $v_{s}$ and $R_{s}$ to a function of the form

$$
f\left(T_{\mathrm{X}}\right)=K\left(\frac{T_{\mathrm{X}}}{7.8 \times 10^{7} \mathrm{~K}}\right)^{\alpha},
$$

as suggested by equations (3) and (4), we have obtained the values for the coefficients $K_{v_{s}}, \alpha_{v_{s}}$ and $K_{R_{s}}, \alpha_{R_{s}}$, respectively, that are reported in Table 1 . Note that $\alpha_{v_{s}}=\alpha_{R_{s}}=0.5$ is expected for both SCDM and $\Lambda$ CDM from scaling relations. Clearly, the expected trends are reproduced only for the shock velocity, whereas strong deviations from equation (4) appear in the plots for $R_{s}$ in both cosmologies (Fig. 5, bottom). The most dramatic are the differences for $\alpha_{R_{s}}$. The small value reported in Table 1 with respect to that in equation (4) suggests that $R_{s}$ is almost independent of the type of cluster. Although numerical errors both in the simulations and in the data analysis must be considered, such discrepancies are probably true and related to the actual structure of the flows. As already described in the previous section, the formation process of GCs imprints complex, irreducible three-dimensional shock structures, which make it difficult to describe each $\mathrm{GC}$ with a single shock radius.

TABLE 1

ACCRETION-Flow BeSt-Fit Parameters

\begin{tabular}{ccccccc}
\hline \hline Model & $\begin{array}{c}K_{v_{s}} \\
\left(10^{3} \mathrm{~km} \mathrm{~s}^{-1}\right)\end{array}$ & $\alpha_{v_{s}}$ & $\begin{array}{c}K_{R_{s}} \\
(\mathrm{Mpc})\end{array}$ & $\alpha_{R_{s}}$ & $\left(10^{45} \mathrm{ergs} \mathrm{s}^{-1}\right)$ & $\alpha_{\Phi}$ \\
\hline SCDM $\ldots \ldots . .$. & 1.8 & 0.47 & 1.75 & 0.1 & 2 & 2.0 \\
$\Lambda$ CDM $\ldots . . .$. & 1.9 & 0.52 & 1.4 & 0.1 & 6.7 & 2.0 \\
\hline
\end{tabular}




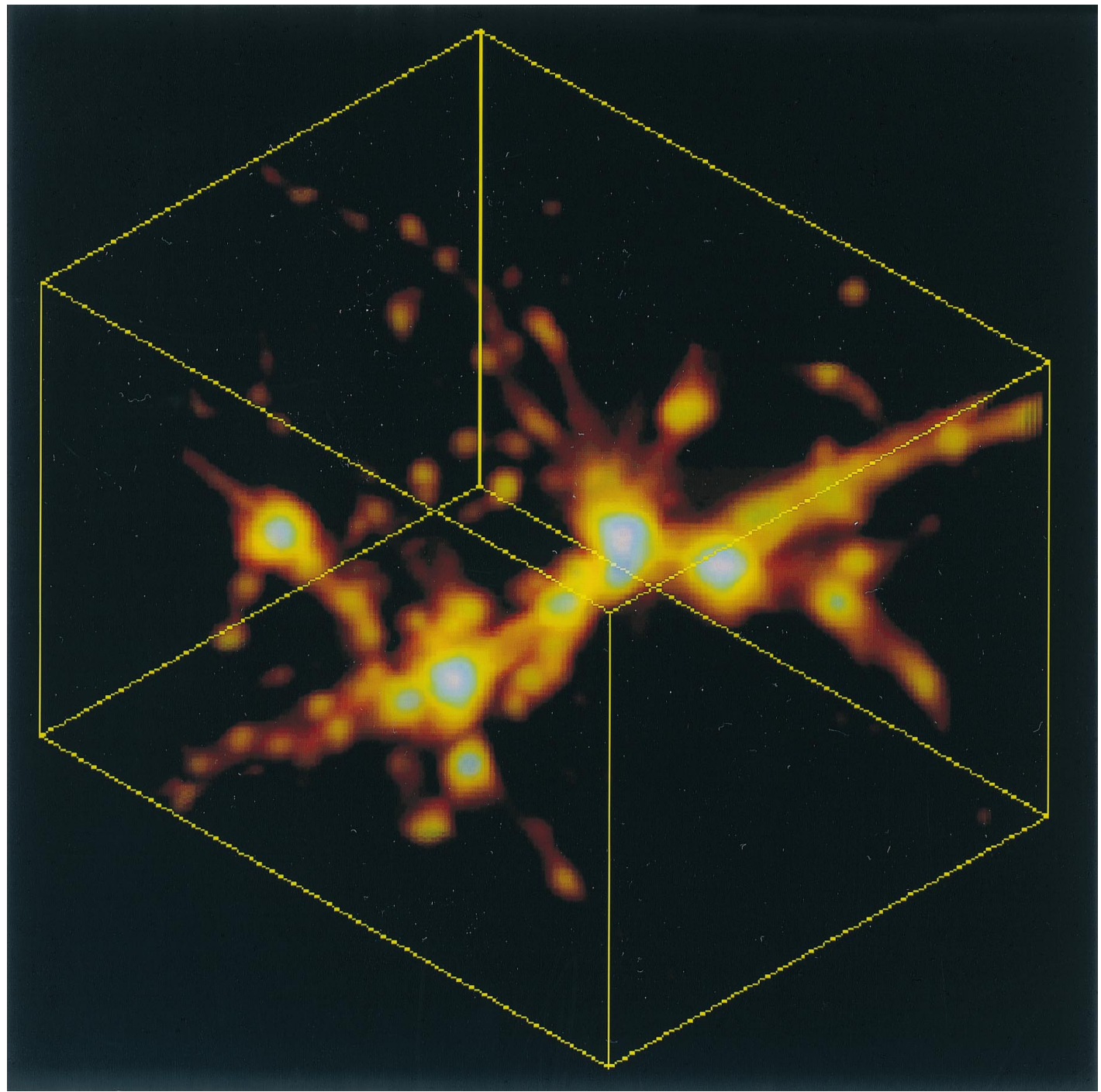

FIG. $3 b$

On the other hand, as long as the thermalization of the accretion kinetic energy takes place, the $v_{s}$ versus $T_{\mathrm{X}}$ relation is less affected by the flow structure, with $\alpha_{v_{s}}$ very close to 0.5 for both cosmologies. Here $K_{v_{s}}$ is consistent with the predictions of self-similar solutions for SCDM as well as $\Lambda \mathrm{CDM}$, for the same reason. We point out that $v_{s}$ is the normal component of the accreting gas in the shock rest frame. This is the component that undergoes dissipation, originating the postshock gas temperature. However, the three-dimensional accretion flow also possesses transverse velocity components that are not thermalized across the shock and that can generate turbulent motions inside the IGM of GCs. In this regard, Eulerian, uniform grid-based schemes may be among the best choices to capture this component of the flow in terms of a balance between resolution and computational performance. In fact, on the one hand in smoothed-particle hydrodynamic (SPH) methods turbulence can be suppressed by excessive viscosity. On the other hand, the higher computational cost paid by the advantage of having a higher resolution with adaptive-mesh refinement techniques is not completely satisfactory because the small scales of the turbulent component are not fully generated.
We have also calculated the flux of kinetic energy across shocks, defined as

$$
\Phi_{E_{k}}=\frac{1}{2} \rho v_{s}^{2} R_{s}^{2} v_{s} .
$$

This quantity is shown in Figure 6 as a function of $T_{\mathrm{X}}$ for the SCDM (left) and $\Lambda$ CDM (right) models. It is of interest in relation to $\mathrm{CR}$ acceleration, because it represents the amount of power available for conversion into suprathermal particles. It provides a large amount of power of the same order as the X-ray luminosity emitted from a central region of $0.5 h^{-1} \mathrm{Mpc}$ (see KCOR94 and CO94 for the amount of the cluster's X-ray luminosity). It is clear that if a modest fraction of this inflowing kinetic energy can be converted into cosmic rays, these may become important sources of emissions (by synchrotron and inverse-Compton scattering) and even play a dynamical role through CR pressure. Here $\Phi_{E_{k}}$ was fitted by a power law of the form

$$
\Phi_{E_{k}}=K_{\Phi}\left(\frac{T_{\mathrm{X}}}{7 \times 10^{7} \mathrm{~K}}\right)^{\alpha_{\Phi}} .
$$

The best-fit parameters are reported in Table 1 . As we can see, the normalization factor, $K_{\Phi}$, is larger for the $\Lambda \mathrm{CDM}$ 

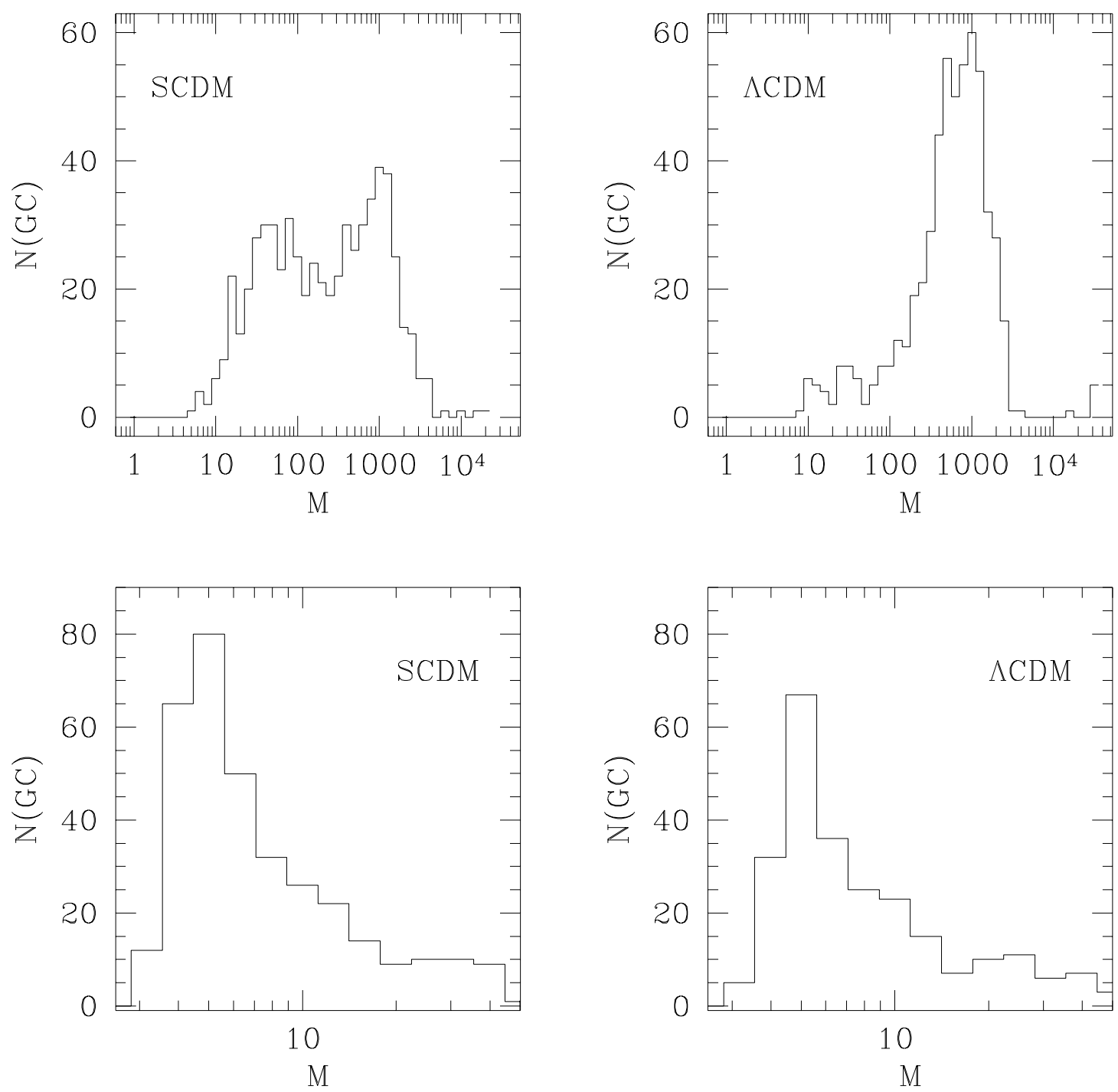

Fig. 4. - Histograms showing the distribution of clusters of galaxies vs. shock Mach number, for external accretion shocks (top) and those inside a region of $0.5 h^{-1} \mathrm{Mpc}$ radius around the cluster center (bottom), and for SCDM (left) and $\Lambda$ CDM (right).

case than for the SCDM one. Moreover, both slopes are larger than the values implied from the slopes of $v_{s}$ and $R_{s}$ in combination with equation (6). This is probably due to an additional dependence of the accreting gas density on the cluster temperature. In fact, $\Phi_{E_{k}}$ is calculated at the first shock cells encountered along the coordinate axis from the GC center (and then averaged over the accretion surface). Since such cells could well be inside "external" shocks, as illustrated in Figures 1 and 2, the density of the accreting gas depends on the properties of the cluster environment. In particular, we know that (1) for a given cosmological model, such density is higher around larger (higher temperature) clusters, implying a steeper increase of $\Phi_{E_{k}}$ than for a temperature-independent density value. (2) In addition, for the same cluster temperature, the corresponding cluster gas density, and therefore $K_{\Phi}$ are larger for a $\Lambda$ CDM model than for a SCDM one. This second point is related to the fact that in general, clusters in $\Lambda \mathrm{CDM}$ have temperatures smaller that those in SCDM (e.g., Figs. 5 and 6; see also KCOR94, CO94).

It turns out that $\Phi_{E_{k}}$ is a steep function of cluster temperature, spanning several orders of magnitude in the tem- perature range of the identified clusters. This means that if the CR acceleration mechanism at shocks around GCs possesses an injection mechanism and an efficiency independent of the cluster properties (e.g., mass and temperature), then we would expect hotter clusters to store a relatively larger amount of nonthermal energy in the form of relativistic particles. Such a trend has already been observed; for instance, Liang (1999) reported a positive correlation between the radio emission and the X-ray temperature in GCs.

\subsection{Evolutionary Trends}

In general, complex shock structures are already present at high redshift. At $z=5-10$, shock waves are well formed and have already developed connections with neighbor clusters or protoclusters. The strength of the shocks is largest around the most massive objects, yet far from uniform. As the evolution advances, mergers occur on all scales, affecting all types of structures, including shocks. As a result, at $z=0$, many filamentary structures have coalesced into larger ones. In addition, the shocks associated with them have become stronger and more uniform, 

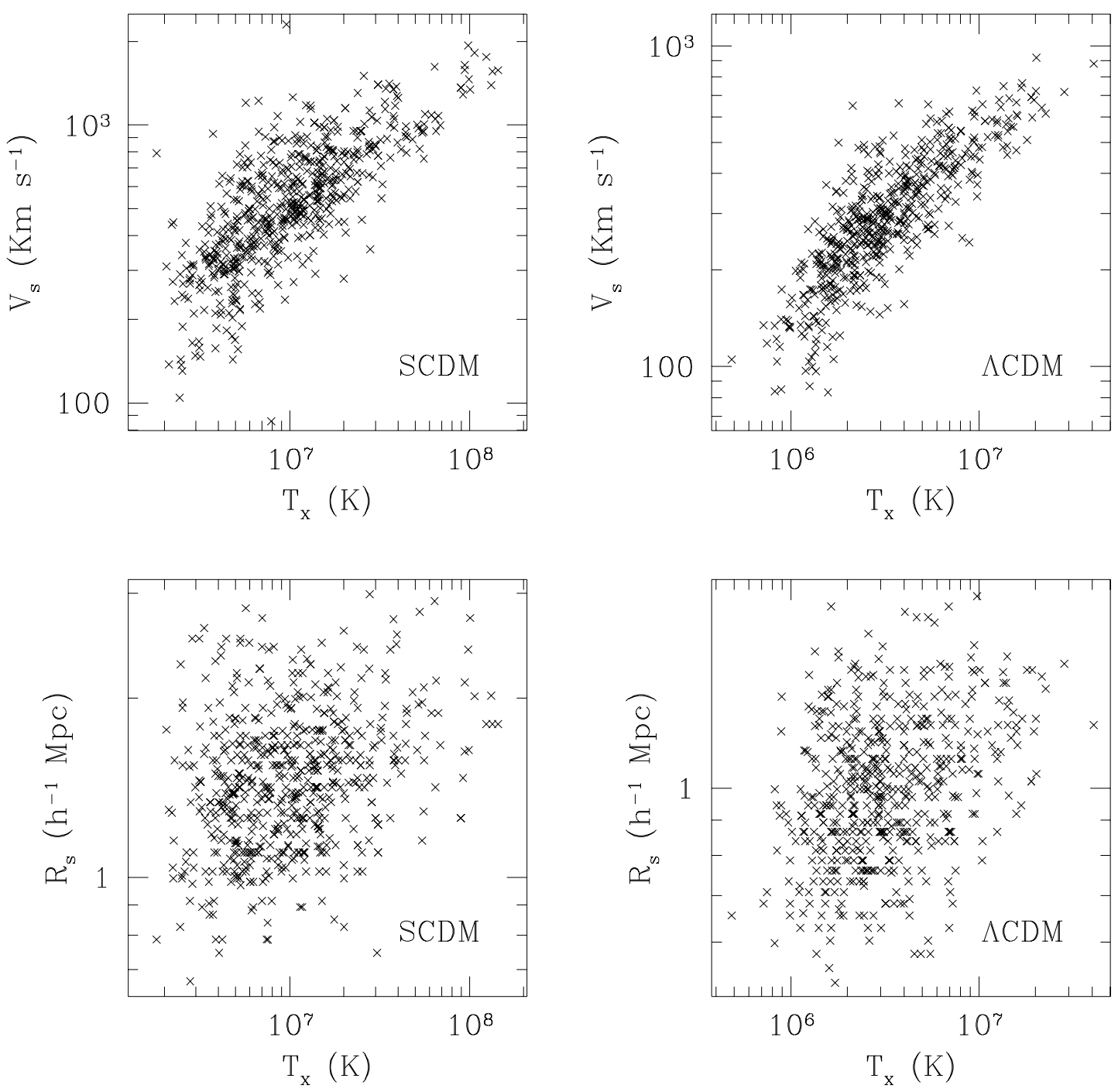

FIG. 5.-Accretion velocity, $v_{s}($ top $)$, and radius, $R_{s}$ (bottom), as a function of cluster core temperature, $T_{\mathrm{X}}$, for $\operatorname{SCDM}($ left $)$ and $\Lambda \mathrm{CDM}($ right $)$

due to the increased amount of matter onto which the gas is being accreted.

In order to study the characteristics of shock evolution, we define the following quantity:

$$
S(z, M)=\frac{1}{N_{\text {tot }}(1+z) d x} \int_{\infty}^{M} \frac{d}{d M^{\prime}}\left[N_{\text {shock }}(z,>M)\right] d M^{\prime},
$$

where $N_{\text {shock }}(z,>M)$ is the number of cells hosting a shock of Mach number greater than $M$ at redshift $z, N_{\text {tot }}$ is the total number of cells in the computational box, and $(1+z) d x$ is the comoving cell size. Figure 7 shows the evolution of $S(z, 1)$, representing the inverse of the average comoving distance between shocks of any Mach number, as a function of redshift $(z)$ for SCDM (open circles) and $\Lambda \mathrm{CDM}$ (filled circles). This quantity also represents the ratio of shock surface over space volume. The following characteristics stand out from this plot. First, it is clear that cosmic plasma was populated with many more shocks in the past than nowadays. Second, while $S(z, 1)$ peaks at $z \simeq 4.6$ in the $\Lambda C D M$ model, we can only say that it peaks at $z \gtrsim 5$ in the SCDM model, given our limited data set.
In addition, in recent epochs $S(z, 1)$ is substantially larger in the $\Lambda$ CDM model than in the SCDM model. Finally, the evolution is smooth in the $\Lambda$ CDM case, but shows abrupt transitions in the SCDM case.

As for the first point made above, the larger area of shock surfaces is due to the extremely filamentary structure of the universe at higher redshifts. Filaments are confined by accretion shocks. As already pointed out in $\S 3.1$, as structure formation progresses, filaments coalesce, therefore growing thicker and rarer. Although the size of their associated shocks increases, their reduced population plays the dominant role in determining overall a decrease of the total area of shock surfaces in a comoving volume.

The second point then tells us that although such a process takes place in both cosmologies, there are many more filaments today in the $\Lambda$ CDM than in the SCDM scenario. This is clearly illustrated in Figure 8, where we show two-dimensional slices, with thickness $0.315 h^{-1} \mathrm{Mpc}$, of the temperature structure of the universe at two different redshifts, $z=3$ (top) and 0 (bottom), for SCDM (left) and $\Lambda \mathrm{CDM}$ (right) models. There we can see that at $z=3$, the two universes look quite similar, with comparable amounts of filaments, in accord with Figure 7. However, at later 

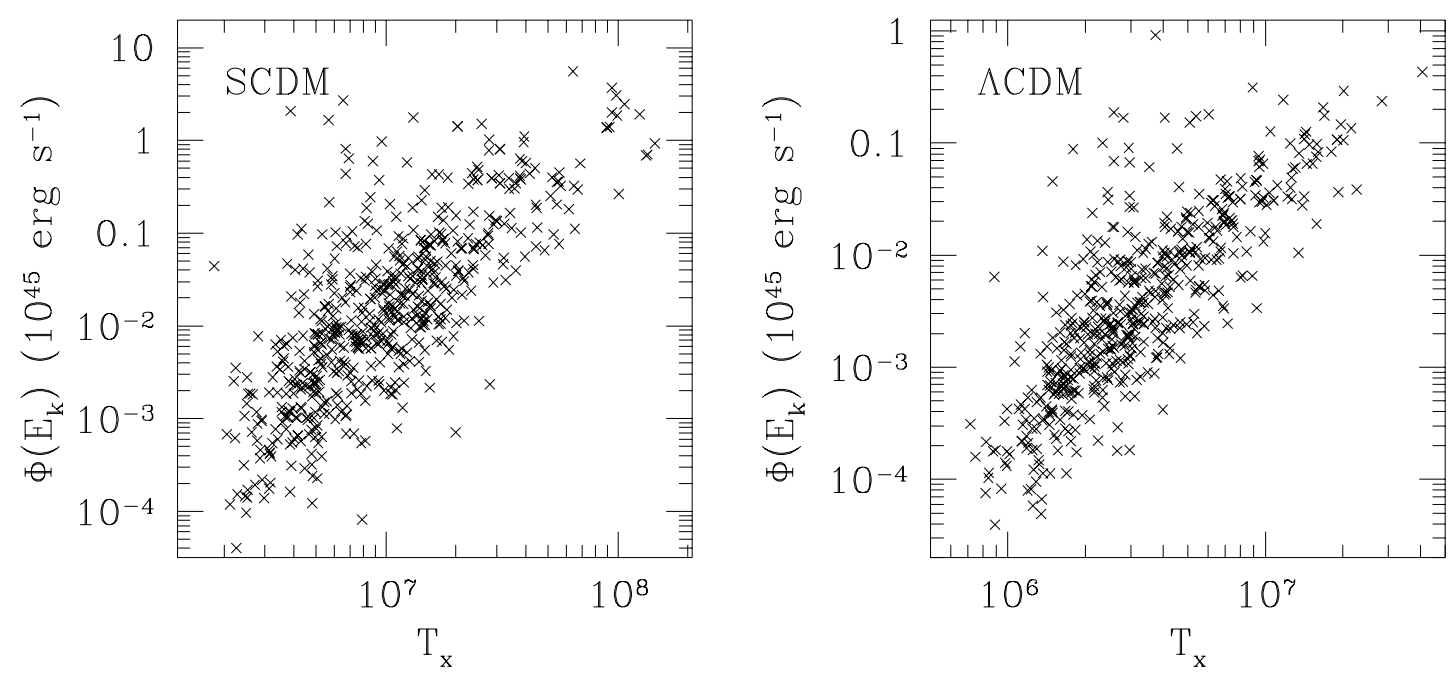

FIG. 6. - Kinetic energy flux across accretion shock, $\Phi_{E_{k}}$, as a function of cluster core temperature, $T_{\mathrm{X}}$, for $\operatorname{SCDM}($ left $)$ and $\Lambda \mathrm{CDM}($ right $)$

times, i.e., $z=0$, filaments in the SCDM model are fatter and rarer as compared to the $\Lambda \mathrm{CDM}$ case. This finding is a reflection of the different initial conditions in the two models. In particular, the larger amplitude of the primordial perturbations used in the SCDM, $\sigma_{8}=1.05$, gives rise, at the current epoch, to a more clustered but less filamentary structure in this model than in $\Lambda$ CDM (cf. KCOR94; CO94).

Turning to the final point, sudden reductions of $S(z, 1)$ are located at $z \simeq 3,0.7$, and 0.2 in SCDM. These reflect the occurrences of a higher rate of merging processes at those particular epochs.

Further details related to the time evolution of shocks are illustrated in Figure 9, where we plot as a function of the

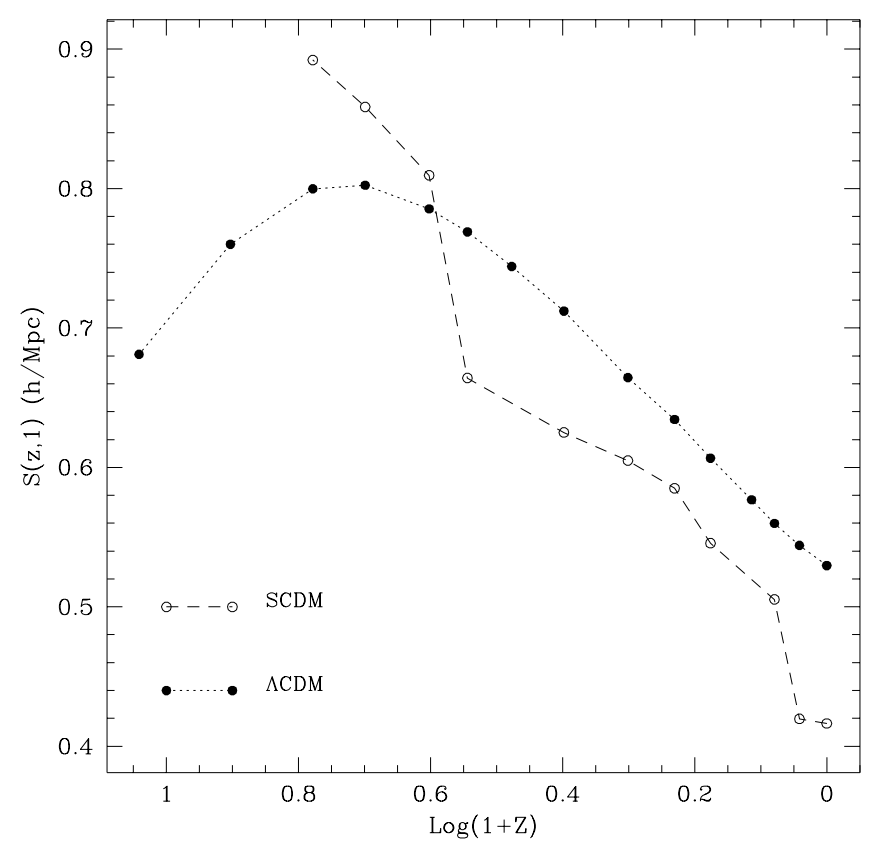

FIG. 7.- $S(z, 1)$, representing the inverse of the average comoving distance between shocks, as a function of cosmological redshift $z$ for SCDM (open circles) and $\Lambda \mathrm{CDM}$ ( filled circles).
Mach number the quantity

$$
W(z, M)=\frac{d}{d z} S(z, M) .
$$

Here $W(z, M)$ expresses the negative of the rate of formation of shocks with Mach number greater than $M$ at a particular epoch $z$. Thus, from Figure 9 we can see that at early times $(z=4.5)$ shocks are forming with Mach number in the range between $\sim 10$ and a few $\times 10^{2}$ for both the $\Lambda \mathrm{CDM}$ and SCDM cases. No shocks exist with $M>$ a few $\times 10^{2}$, and for $M<10$ shocks are being depleted due to merger events. Later on, at $z=1.25$, shocks start forming in the range $10^{2} \lesssim M \lesssim 10^{3}$. In accord with Figure 7, the total rate of shock formation $[\equiv W(z, 1)]$ is always negative. As already pointed out, the numerous weak-filament shocks are replaced by stronger but rarer shocks. The last two panels $(z=0.6$ and 0.05$)$ show the smooth shock-formation evolution in the $\Lambda$ CDM. A small amount of shock formation still occurs for high Mach numbers, but overall the shock population is decreasing at an increasing rate. The situation is more complex in the SCDM case. At $z=0.6$, identified above as an epoch of high merging rate, shocks are reduced at any Mach number. At $z=0.05$, the largest Mach number shocks $\left(M \gtrsim 5 \times 10^{2}\right)$ are depleted, indicating merging of the most massive objects.

In order to assess the relative importance of shocks at different epochs in terms of CR contribution, in Figure 10 we plot the adimensional quantity

$$
F(z)=\frac{\left[\Phi_{E_{k}}(z)\right]_{\text {shock }}}{\left(\rho_{c} H_{0}^{3} \lambda_{\text {n1 }}^{5}\right)_{z=0}},
$$

where $\Phi_{E_{k}}$ is the total flux of kinetic energy through shocks (of any Mach number), $\rho_{c}$ is the critical density, $H_{0}^{3}$ is the Hubble constant, and $\lambda_{n 1}$ is the nonlinear perturbation wavelength, set to $50 h^{-1} \mathrm{Mpc}$. Figure 10 shows that the flux of kinetic energy through shocks today has increased by a few orders of magnitude with respect to early epochs, say $z \simeq 5$. Thus, today's shocks retain more kinetic energy than ever. However, the time-integrated flux of kinetic energy through shocks (e.g., since $z=5$ ) is much larger than the thermal energy content at $z=0$, which, according to 

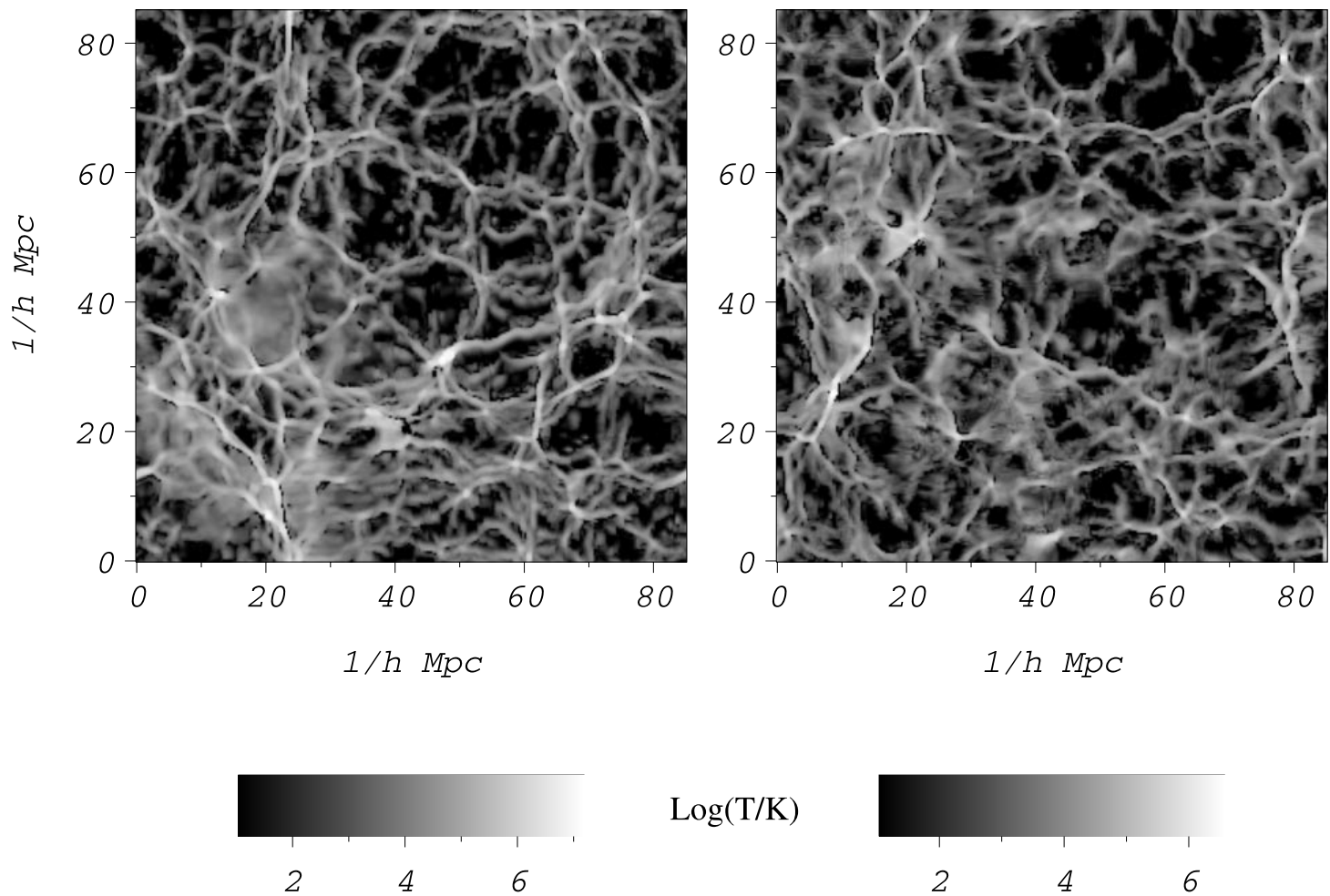

$\log (\mathrm{T} / \mathrm{K})$
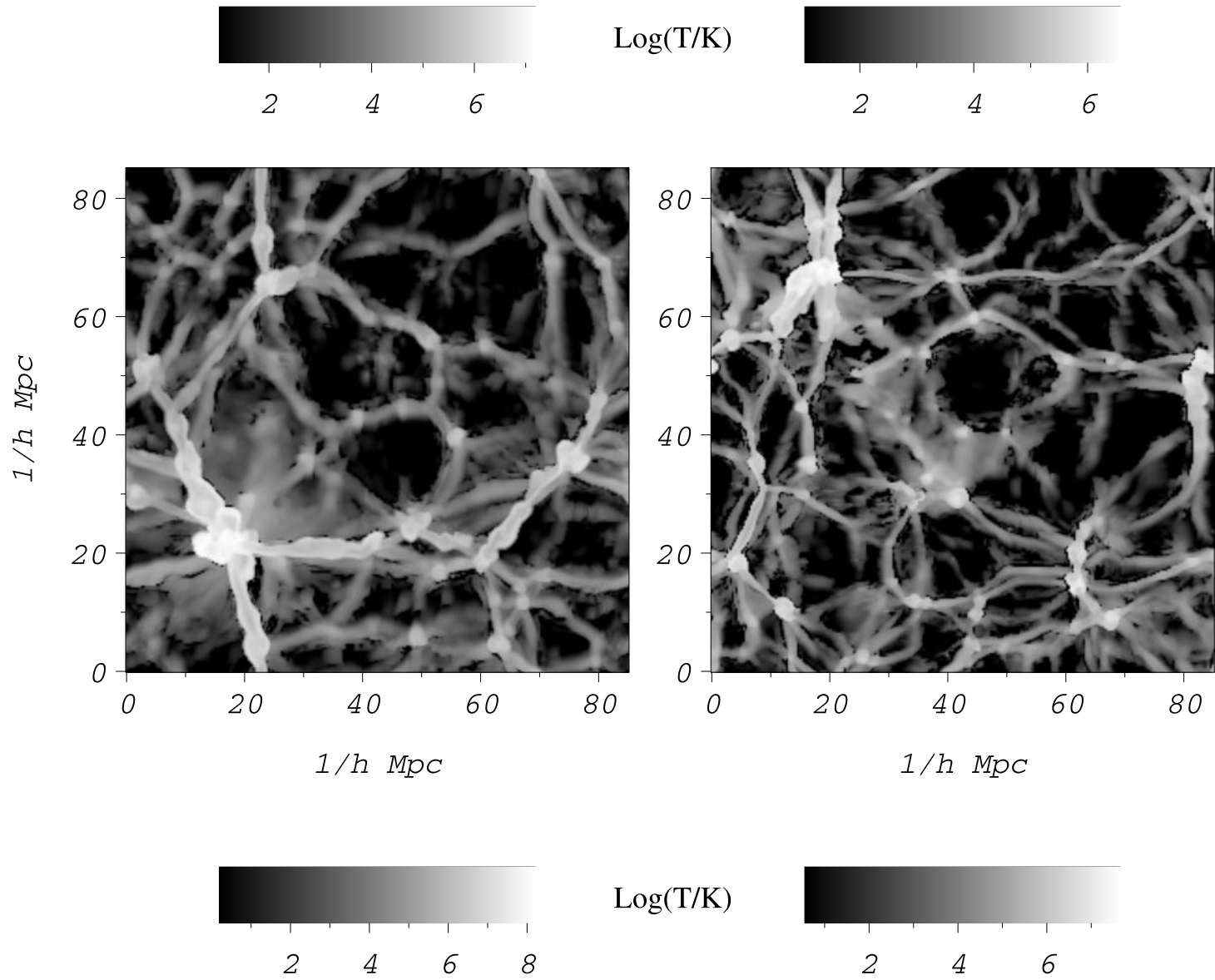

$\log (\mathrm{T} / \mathrm{K})$

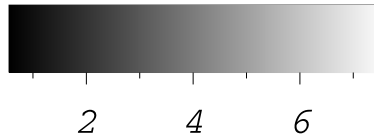

FIG. 8. - Slices of temperature distribution showing the filamentary structure in SCDM $(l e f t)$ and $\Lambda$ CDM (right) models at cosmological redshift $z=3$ (top) and 0 (bottom).

our data, has mostly been produced after $z \sim 1.5$. This is not due to the low thermalization efficiency of shocks. Rather, that is because although shocks form at much higher redshifts, the thermal energy they produce undergoes severe adiabatic losses due to cosmological expansion. On the other hand, after roughly $z \sim 1.5$, such thermal energy is retained inside well-formed structures such as clusters and filaments.
In order to identify the characteristics of the most relevant shocks, i.e., those that process most of the gas, we calculate

$$
Y(M)=\frac{1}{E_{\mathrm{th}}(z=0)} \int_{t(z=1.5)}^{t(z=0)} \frac{d \Phi_{E_{k}}(M)}{d \log M} d t^{\prime},
$$

where the limits of integration have been chosen on the basis of the arguments in the previous paragraph. Here, 

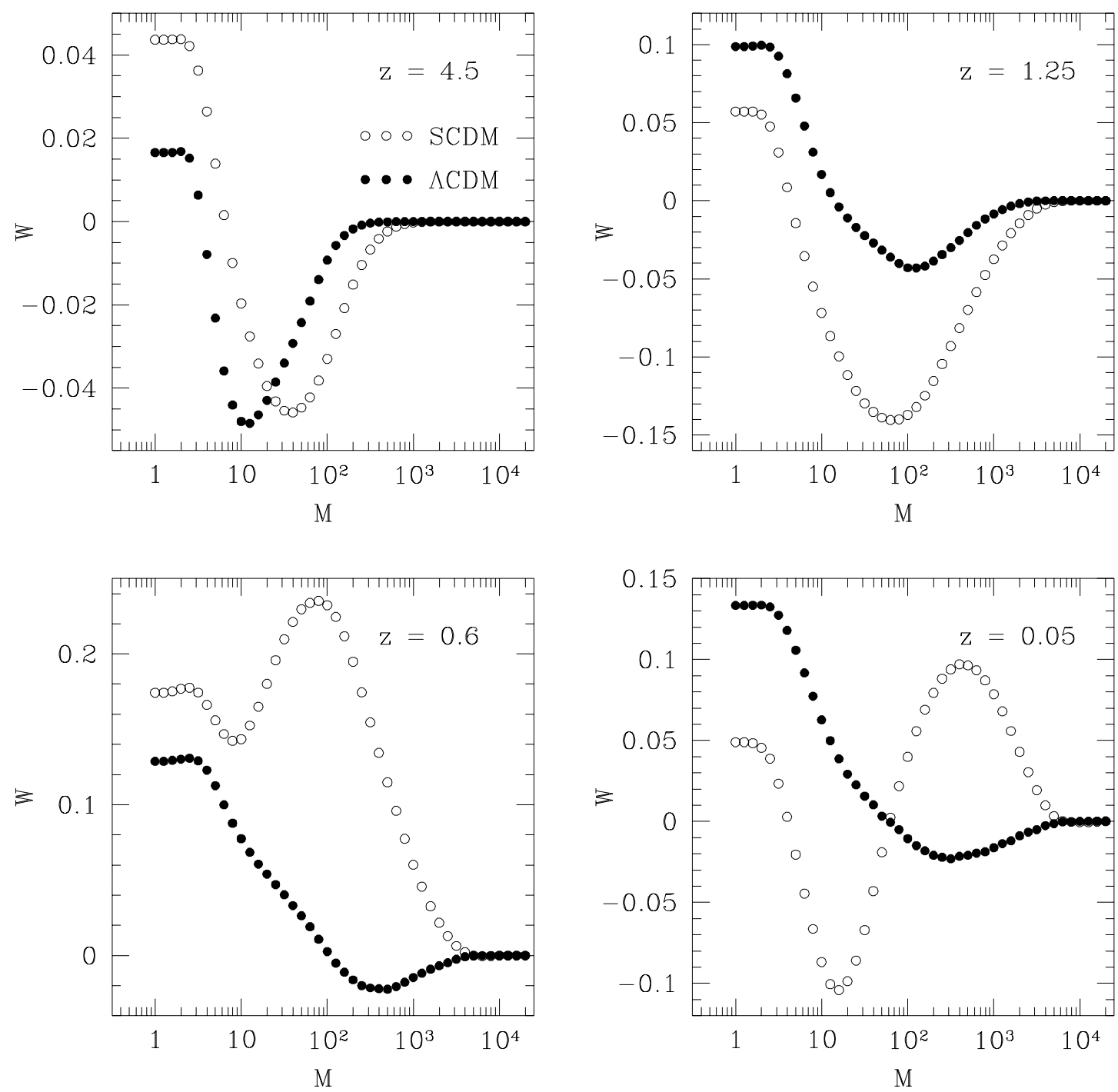

FIG. 9.- Redshift derivative of the fraction of shocks with Mach number greater than $M$ as a function of $M$. Note that since $d t \propto-d z$, positive values of $W$ indicate a decreasing population of shocks.

$\Phi_{E_{k}}(M)$ is the kinetic energy flux through shocks with Mach number between $\log M$ and $\log (M+d M)$, and $E_{\mathrm{th}}(z=0)$ is the total thermal energy inside the computational box at $z=0$. This quantity is plotted in Figure 11 as a function of Mach number $M$ for the SCDM (left) and $\Lambda$ CDM (right) models.

We can see that most of the flux of kinetic energy occurs through shocks with ("low") Mach numbers around 4, which correspond to our internal shocks. In fact, although most of the shocks have Mach numbers much larger than that (see histograms of Fig. 4), low Mach number shocks are typically located inside much denser regions (formed structure) and therefore process much more matter and kinetic energy than average (as already pointed out in $\S 3.2$, the gas velocities for internal and external shocks are comparable). This depiction is not just a result of the integration. A more detailed analysis of plots of $d Y(M) / d t$, describing the flux of kinetic energy as a function of $M$ for different redshifts (not shown here) shows that the qualitative features of the curve in Figure 11 are common at any $z$, and therefore the low Mach number shocks are always responsible for most of the processing of the kinetic energy of the peculiar motions.

Finally, integration of the area underneath each curve in Figure 11 represents the total kinetic energy passed through shocks since $z=1.5$ divided by the thermal energy at $z=0$. Its value is $\sim 17$ for the SCDM and $\sim 13$ for the $\Lambda$ CDM models, respectively. If a fraction $\epsilon \sim 10^{-2}$ of such energy is transferred to $\mathrm{CR}$ protons, then the energy stored up in cosmic rays today should amount to about $15 \%$ of the thermal energy inside formed structure. This is only a rough estimate, which needs to be refined by more accurate calculations.

\section{DISCUSSION}

We have studied the properties of cosmic shock waves associated with the large-scale structure of the universe in two different cosmological scenarios, namely, SCDM and $\Lambda$ CDM. Such shocks reveal remarkable properties. In fact, hierarchical formation histories of GCs produce highly complex flows and shock structures, which extend over scales of several Mpc. In addition to accretion shocks 


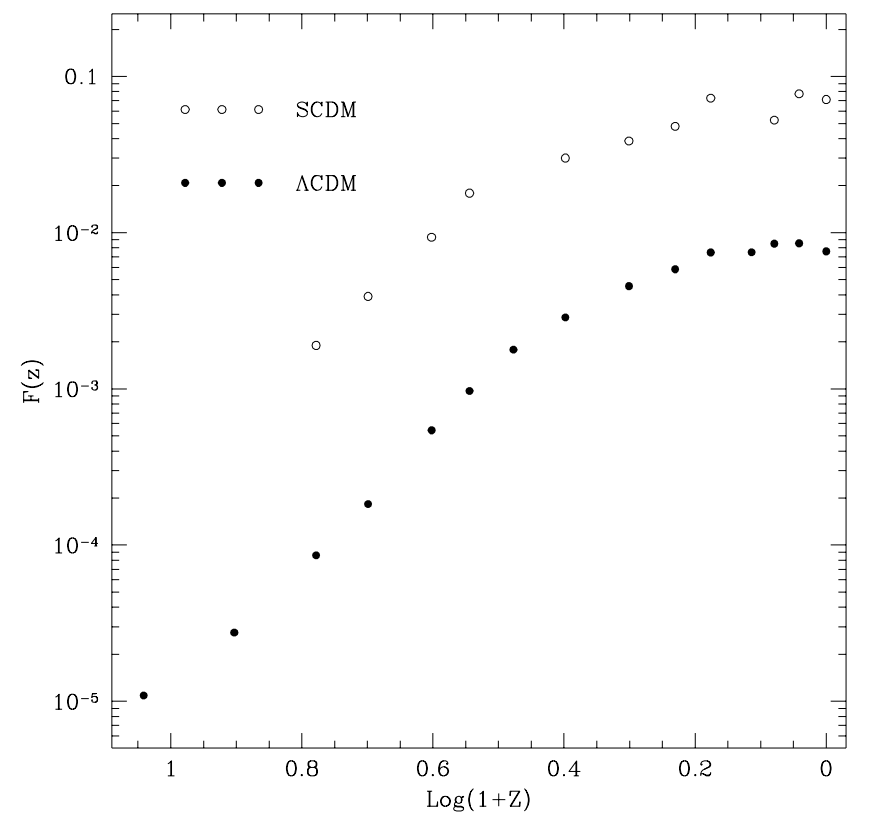

FIG. 10.-Evolution of $F(z)$, representing the total kinetic energy flux through shocks of any Mach number, normalized to $\rho_{c} H_{0}^{3} \lambda_{\mathrm{n} 1}^{5}$. Here we set $\lambda_{\mathrm{n} 1}=50 \mathrm{~h}^{-1} \mathrm{Mpc}$. Open circles are for the SCDM, and filled circles for the $\Lambda \mathrm{CDM}$ case.

(responsible for heating infalling gas) and merger shocks, flow shocks also appear and propagate through the thermalized ICM, providing extra gas heating. It turns out that the morphology of shocks associated with a large-scale structure is complex and irreducibly three-dimensional, and spherical shapes are inadequate to their description. Only for the external accretion shocks, located far away from the cluster core, is some form regularity recovered. This is an important issue, especially from the perspective of missions with the next generation of high-resolution X-ray telescopes (Chandra and $X M M$ ) that are planning to detect shocks in the ICM. It is worth mentioning that the work of Ensslin et al. (1998) might already provide observational evidence for the presence of flow shocks in cosmic structure. Their con-

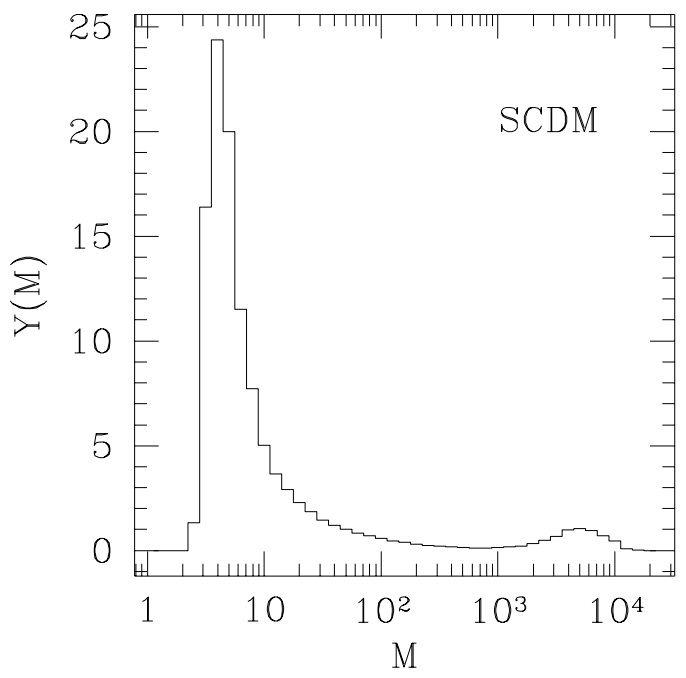

clusions are based on the assumption that the observed radio emission is due to particles currently accelerated at shocks there and injected from a "radio relic," a remnant previously associated with some radio galaxies. For example, for $1253+275$, they find a preshock gas temperature of $T \sim 0.5-1 \mathrm{keV}$, which shows that this is not the case of an accretion shock, but either a merger or a flow shock (propagating through the ICM). Since there is no evidence for a merging process in $1253+275$, it must be a flow shock. In addition, from their reported data on the pressure jump, we infer $M \sim 3-4.2$, well within the expected range for the internal shocks shown in Figure 4 (bottom).

Cosmic shocks are also ideal sites for particle acceleration. We have shown already in $\S 3.2$ that cosmic shocks provide enough power to produce copious cosmic rays. The details of the produced populations will depend on the injection mechanism and scattering agent, i.e., the magnetic field and the diffusion properties. Here we further stress the important role of merger and flow shocks. These shocks may be responsible not only for the acceleration of cosmic rays out of the thermal pool of the ICM, but also for the reacceleration of cosmic rays produced at accretion shocks and/or ejecta from radio galaxies, active galactic nuclei, or normal galaxies. In addition, they could be crucial in terms of acceleration and transport of ultra high energy cosmic rays, because the scattering mean free path of these particles is of the same order as the typical separation between accretion, merger, and flow shocks in the ICM. As already pointed out at the beginning of this section, there seems to be a solid foundation for the existence of such shocks.

As described in $\S 1$, the presence of relativistic CR electrons in GCs has been inferred through observations of diffuse synchrotron radiation from radio halos. In addition, there is evidence for an excess of radiation in both the EUV and hard X-ray bands with respect to thermal emission. Although still of controversial interpretation, such excesses are probably due to inverse-Compton emission of CR electrons scattering off cosmic microwave background photons. Published studies, however, reveal that for an accurate interpretation of the constraints from the combined nonthermal emission components, it is crucial to have a

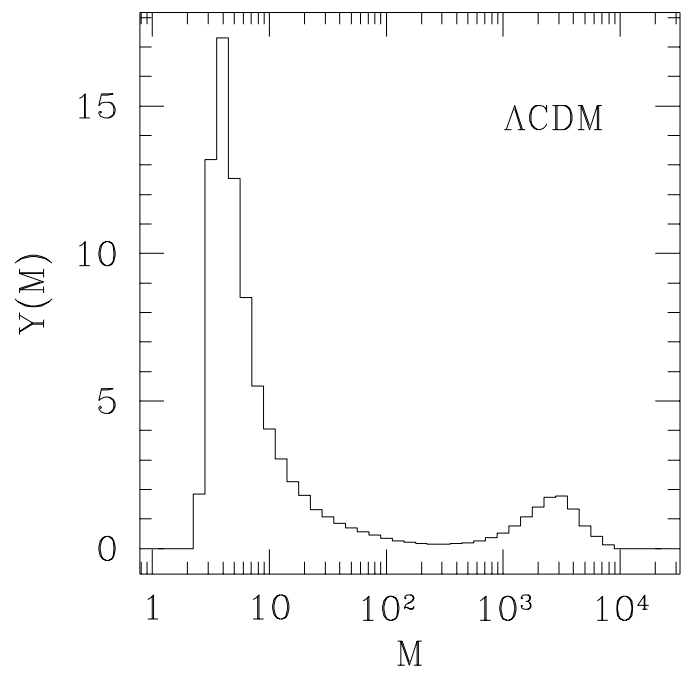

FIG. 11.-Time-integrated amount of kinetic energy passed thorough shocks with Mach number between $\log M$ and $\log (M+d M)$ since the epoch at $z=1.5$, normalized to the total thermal energy inside the computational box at $z=0$ and divided by $d \log M(=0.1)$, as a function of Mach number $M$. Plots are for SCDM (left) and $\Lambda$ CDM (right). 
detailed depiction of the relative distribution of particles and magnetic fields (Ensslin et al. 1999).

The proton component of cosmic rays has not been directly observed. Nonetheless, given the estimates for the CR electron component, Lieu et al. (1999) concluded that their contribution in terms of dynamical pressure in GCs could be comparable to the thermal gas. This is consistent with the estimate inferred in the previous section and, as already pointed out in the introduction, has important consequences for cosmology. We point out that cosmic shock waves have existed ever since nonlinear structure formation was initiated at high redshift. This was shown through Figure 7 in $\S 3.3$. Therefore, the importance of nonthermal activities in the cosmic plasma traces back to early epochs. Collisions of CR proton in the ICM generate, however, a flux of gamma-ray photons through the production and subsequent decay of neutral pions; as pointed out by Blasi (1999), such gamma-ray flux seems to be only marginally compatible with the upper limits measured by EGRET for the Coma and Virgo Clusters. Again, however, the spatial and spectral distributions of cosmic rays, both depending on the overall cosmological history of these particles, play a crucial role in determining the expected gamma-ray flux. In any case, the advent of the new generation of gamma-ray facilities (GLAST, VERITAS) characterized by a much higher sensitivity (cf. Blasi 1999 for more details) will definitely settle the issue.

From this depiction emerges the importance and the necessity of understanding the role of cosmic rays in cosmology. For this purpose we are developing numerical tools in order to consistently treat magnetic fields and cosmic rays in numerical simulations of structure formation. Such tools, in fact, will allow us to explicitly follow the evolution of the magnetic field as well as the acceleration and transport of cosmic rays. With this information, we will be able to carry out very useful comparisons between numerical and observational results in various bands of the electromagnetic spectrum.

F. M. was supported by a doctoral dissertation fellowship at the University of Minnesota. F. M. and T. W. J. were supported in part by NSF grants AST 96-16964 and INT 95-11654, NASA grant NAGS-5055, and by the Minnesota Supercomputing Institute. D. R. and H. K. were supported in part by grant 1999-2-113-001-5 from the interdisciplinary Research Program of the KOSEF. R. C. and J. P. O. were supported in part by NSF grants AST 98-03137 and ASC 97-40300.
Arnaud, M. 1994, in Cosmological Aspects of X-Ray Clusters of Galaxies, ed. W. C. Seitter (Dordrecht: Kluwer), 197

Bahcall, N. A., \& Fan, X. 1998, ApJ, 504, 1

Bertschinger, E. 1985, ApJS, 58, 39

Blandford, R. D., \& Ostriker, J. P. 1978, ApJ, 221, L29

. 1980, ApJ, 237, 793
Blasi, P. 1999, ApJ, 525, 603

Blasi, P., Burles, S., \& Olinto, A. 1999, ApJ, 514, L79

Carlberg, R. G., Morris, S. L., Yee, H. K. C., \& Ellingson, E. 1997, ApJ, 479, L19

Cen, R., \& Ostriker, J. P. 1994, ApJ, 429, 4 (CO94) . 1999a, ApJ, 514, 1 1999b, ApJ, 517, 31

Chandran, B. D. G. 1997, ApJ, 485, 148

Deiss, B. M., Reich, W., Lesch, H., \& Wielebinsky, R. 1997, A\&A, 321, 55

Donnelly, R. H., Markevitch, M., Forman, W., Jones, C., Churazov, E., \& Gilfanov, M. 1999, ApJ, 513, 690

Ensslin, T. A., Biermann, P. L., Klein, U., \& Kohle, S. 1998, A\&A, 332, 395

Ensslin, T. A., Biermann, P. L., Kronberg, P., \& Wu, X.-P. 1997, ApJ, 477, 560

Ensslin, T. A., Lieu, R., \& Biermann, P. L. 1999, A\&A, 344, 409

Evrard, A. E. 1990, ApJ, 363, 349 1997, MNRAS, 292, 280

Evrard, A. E., Metzler, C. A., \& Navarro, J. F. 1996, ApJ, 469, 494

Fabian, A. C. 1996, Science, 271, 1244

Frenk, C. S., et al. 1999, ApJ, 525, 554

Fusco-Femiano, R., Dal Fiume, D., Feretti, L., Giovannini, G., Grandi, P., Matt, G., Molendi, S., \& Santangelo, A. 1999, ApJ, 513, L21

Giovannini, G., Feretti, L., Venturi, T., Kim, K. T., \& Kronberg, P. P. 1993, ApJ, 406, 399

Henriksen, M. 1998, PASJ, 50, 389

Jones, F. C., \& Ellison, D. C. 1991, Space Sci. Rev., 58, 259

Kaastra, J. 1998, in Proc. 32d COSPAR Scientific Assembly (Paris: CNS), in press

Kang, H., Cen, R., Ostriker, J. P., \& Ryu, D. 1994a, ApJ, 428, 1 (KCOR94)

\section{EFERENCES}

Kang, H., Ostriker, J. P., Cen, R., Ryu, D., Hernquist, L., Evrard, A. E., Bryan, G. L., \& Norman, M. L. 1994b, ApJ, 430, 83

Kang, H., Rachen, J. P., \& Biermann, P. L. 1997, MNRAS, 286, 257

Kang, H., Ryu, D., \& Jones, T. W. 1996, ApJ, 456, 422

Kim, K.-T., Kronberg, P. P., Giovannini, G., \& Venturi, T. 1989, Nature, 341,720

Kronberg, P. P. 1994, Rep. Prog. Phys., 325, 382

Kulsrud, R. M., Cen, R. Y., Ostriker, J. P., \& Ryu, D. 1997, ApJ, 480, 481

Lacey, C., \& Cole, S. 1994, MNRAS, 271, L676

Landau, L. D., \& Lifshitz, E. M. 1997, Fluid Mechanics (Oxford: Pergamon)

Liang, H. 1999, in Proc. Ringberg Workshop on Diffuse Thermal and Relativistic Plasma in Galaxy Clusters, ed. H. Boehringer, L. Feretti, \& P. Schuecker (Garching: Max-Planck Institute), 33

Lieu, R., Ip, W.-H., Axford, I. W., \& Bonamente, M. 1999, ApJ, 510, L25

Lieu, R., Mittaz, J. P. D., Bowyer, S., Lockman, F. J., Hwang, C.-Y., \& Schmitt, J. H. M. M. 1996, ApJ, 458, L5

Markevitch, M., Forman, W. R., Sarazin, C. L., \& Vikhlinin, A. 1998, ApJ, 503,77

Mittaz, J. P. D., Lieu, R., \& Lockman, F. J. 1998, ApJ, 498, L17

Molendi, S., De Grandi, S., Fusco-Femiano, R., Colafrancesco, S., Fiore, F., Nesci, R., \& Tamburelli, F. 1999, ApJ, 525, L73

Mushotzky, R. F., \& Scharf, C. A. 1997, ApJ, 482, L13

Navarro, J. F., Frenk, C. S., \& White, S. D. M. 1995, MNRAS, 275, 720

Ostriker, J. P., \& Cen, R. 1996, ApJ, 464, 27

Quilis, V., Ibanez, J. M. A., \& Saez, D. 1998, ApJ, 502, 518

Ryu, D., \& Kang, H. 1997, MNRAS, 284, 416 (RK97)

Ryu, D., Kang, H., \& Biermann, P. L. 1998, A\&A, 335, 19

Ryu, D., Ostriker, J. P., Kang, H., \& Cen, R. 1993, ApJ, 414, 1

Sarazin, C. L. 1999, ApJ, 520, 529

Sarazin, C. L., \& Lieu, R. 1998, ApJ, 494, L177

Suginohara, T., \& Ostriker, J. P. 1998, ApJ, 507, 16

Valinia, A., Henriksen, M., Loewenstein, M., Roettiger, K., Mushotzky, R. F., \& Madejski, G. 1999, ApJ, 515, 42

White, S. D. M., Efstathiou, G., \& Frenk, C. S. 1993, MNRAS, 262, 1023 